\title{
Risk of stroke early after implantation of a left ventricular assist device
}

Takaaki Samura, MD, ${ }^{\text {a }}$ Daisuke Yoshioka, MD, PhD, ${ }^{\text {a }}$ Koichi Toda, MD, PhD, ${ }^{\mathrm{a}}$

Ryoto Sakaniwa, MD, ${ }^{\mathrm{b}}$ Mikito Shimizu, MD ${ }^{\mathrm{c}}$ Shigeru Miyagawa, MD, PhD, ${ }^{\mathrm{a}}$

Yasushi Yoshikawa, MD, PhD, ${ }^{\mathrm{a}}$ Hiroki Hata, MD, PhD, ${ }^{\text {a }}$ Shunsuke Saito, MD, PhD, ${ }^{\text {a }}$

Yasushi Sakata, MD, PhD, ${ }^{\mathrm{d}}$ and Yoshiki Sawa, $\mathrm{MD}, \mathrm{PhD}^{\mathrm{a}}$

\section{ABSTRACT}

Objective: Stroke is one of the major adverse events after left ventricular assist device implantation. Risk of stroke is the highest immediately after left ventricular assist device implantation and then increases again in chronic periods. There is no study that has analyzed risk factors for stroke in acute phase. We investigated the risk factors for stroke in the acute phase after left ventricular assist device implantation in the present study.

Methods: Between October 2005 and December 2016, 158 consecutive patients (mean age, $43 \pm 14$ years; $34 \%$ were women) underwent continuous-flow left ventricular assist device (50 HeartMate II [Abbott Medical, Abbott Park, Ill], 38 DuraHeart [Terumo Heart, Ann Arbor, Mich], 33 Jarvik2000 [Jarvik Heart, New York, NY], 23 EVAHEART [Sun Medical, Moriyama City, Japan], 14 HeartWare [Framingham, Mass]) implantation in our institution. We analyzed the risk factors for a symptomatic stroke within 90 days after left ventricular assist device implantation.

Results: Stroke occurred in 28 patients in the acute phase after left ventricular assist device implantation. Multivariate analysis revealed that low cardiac output (odds ratio, $0.25 ; 0.07-0.92 ; P=.024$ ) during postoperative 12 to 24 hours was the only independent risk factor for stroke in the acute phase. Patients with stroke in the acute phase had higher serum lactate dehydrogenase levels at any point until postoperative 14 days. Patients with the HeartMate II device particularly showed a statistically significant negative relationship between cardiac output during postoperative 12 to 24 hours and serum lactate dehydrogenase levels at postoperative 14 days $(\mathrm{r}=-0.313, P=.03)$.

Conclusions: Our study demonstrated that patients with perioperative lower cardiac output and higher lactate dehydrogenase level developed stroke in the acute phase after left ventricular assist device implantation. These results suggested that maintenance of sufficient left ventricular assist device flow is important in prevention of stroke, which may be related to subclinical pump thrombosis. (J Thorac Cardiovasc Surg 2019;157:259-67)

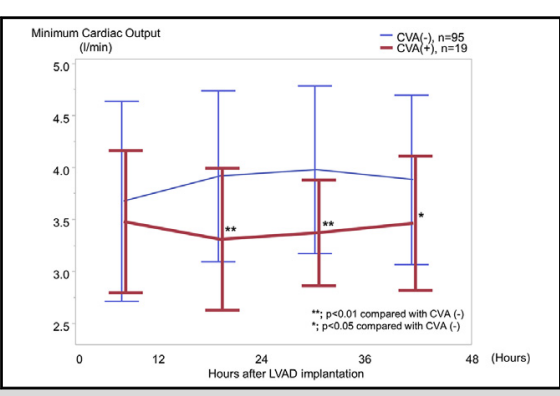

Postoperative course of the minimum cardiac output in patients with stroke versus stroke-free patients.

\section{Central Message}

Perioperative lower cardiac output is strongly related to stroke in the acute phase after LVAD implantation.

\section{Perspective}

Patients with perioperative lower cardiac output and higher LDH level developed stroke in the acute phase after LVAD implantation. These results suggested that maintenance of sufficient LVAD flow plays a critical role in prevention of stroke, which may be related to subclinical pump thrombosis.

See Editorial Commentary page 268

\footnotetext{
From the Departments of ${ }^{\mathrm{a} C}$ ardiovascular Surgery, ${ }^{\mathrm{b}}$ Public Health, ${ }^{\mathrm{c}}$ Neurology, and

${ }^{\mathrm{d}}$ Cardiology, Osaka University Graduate School of Medicine, Osaka, Japan.

Received for publication Dec 13, 2017; revisions received May 24, 2018; accepted for publication June 6, 2018; available ahead of print July 27, 2018.

Address for reprints: Yoshiki Sawa, MD, PhD, Department of Cardiovascular Surgery, Osaka University Graduate School of Medicine, Yamadaoka, 2-2, Suita City, Osaka 565-0871, Japan (E-mail: sawa-p@surg1.med.osaka-u.ac.jp). $0022-5223 / \$ 36.00$

Copyright (C) 2018 by The American Association for Thoracic Surgery https://doi.org/10.1016/j.jtcvs.2018.06.031
}

The evolution of implantable continuous-flow left ventricular assist devices (LVADs) has resulted in the prevailing treatment option for patients with end-stage heart failure. ${ }^{1,2}$ Although a continuous-flow LVAD is associated with a lower

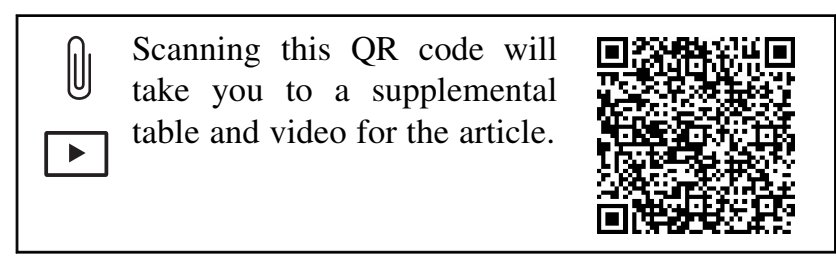




\section{Abbreviations and Acronyms \\ aPTT = activated partial thromboplastin time \\ $\mathrm{CT}=$ computed tomography \\ INR = international normalized ratio \\ $\mathrm{LDH}=$ lactate dehydrogenase \\ LVAD $=$ left ventricular assist device \\ PT-INR $=$ prothrombin time international normalized ratio}

rate of stroke than the older pulsatile LVAD, stroke remains a major adverse event. ${ }^{3,4}$ A previous report revealed that the risk for stroke after LVAD implantation showed a bimodal reversed J-shaped curve with the highest risk immediately after LVAD implantation, which then increases again at postoperative 9 to 12 months. ${ }^{5}$ This result implies that the cause of stroke should be divided into 2 phases: the acute phase and the chronic phase. We have previously revealed that systemic bacteremia was an independent risk factor for hemorrhagic stroke in the chronic phase. ${ }^{4}$ We have also reported that age was an independent risk factor for stroke in the acute phase. ${ }^{4}$ However, in the previous study, we were not able to include data on postoperative hemodynamic and laboratory parameters in data analysis; therefore, we could not demonstrate how to perform postoperative management in the acute phase to prevent stroke. In the present study, we analyzed the risk factors for stroke after LVAD implantation in the acute phase, along with postoperative hemodynamic parameters and laboratory parameters.

\section{MATERIALS AND METHODS \\ Patients}

The ethical committee of our hospital approved this study. Between January 2005 and December 2016, 158 patients (mean age, $43 \pm 14$ years, 53 women) underwent continuous-flow LVAD implantation for end-stage heart failure at Osaka University Hospital. We evaluated the incidence of stroke in these patients and analyzed the risk factors for symptomatic stroke within 90 days after LVAD implantation. All patients and their family members provided informed consent to participate in related clinical studies before LVAD implantation.

\section{Devices and Surgical Procedures}

The type of LVAD used in each patient was decided at the preoperative conference, although available devices have changed over the years in Japan. Briefly, the DuraHeart (Terumo Heart, Ann Arbor, Mich) and EVAHEART (Sun Medical, Moriyama City, Japan) were approved in October 2011, the HeartMate II (Abbott Medical, Abbott Park, Ill) was approved in April 2013, and the Jarvik2000 (Jarvik Heart, New York, NY) was approved in May 2014, whereas the HeartWare (HeartWare, Framingham, Mass) is presently available only for clinical trial use. Before 2010, the DuraHeart and Jarvik2000 were primarily used at Osaka University in a clinical trial subsidized by the Ministry of Health and Labor of Japan.

A detailed method of continuous-flow LVAD implantation has been described. ${ }^{6,7}$ Generally, patients underwent LVAD implantation thorough a median sternotomy with a systemic cardiopulmonary bypass, during which the inflow cannula was inserted into the apex of the left ventricle and the outflow graft was anastomosed to the ascending aorta. Three patients underwent Jarvik2000 implantation through a left thoracotomy with a descending aorta anastomosis of the outflow graft because of the previous cardiac surgery. In patients with mild or greater aortic insufficiency, aortic valve closure with bovine pericardial patch or replacement with a tissue valve was performed, whereas mitral valve repair was done in patients with severe functional mitral regurgitation at the discretion of the surgeons. We typically perform a tricuspid valve procedure in cases with moderate or greater tricuspid regurgitation. The decision regarding concomitant right ventricular assist device insertion was made individually for each patient by the attending surgeons.

\section{Anticoagulant and Medical Therapy During Left Ventricular Assist Device Support}

After LVAD implantation, all patients received a standardized heart failure prevention medical regimen, including neurohormonal antagonists, diuretics, and antiarrhythmic agents, as needed. Aspirin (100 mg daily) monotherapy was also started from the day after LVAD implantation and was maintained throughout LVAD support, unless major bleeding occurred. Anticoagulation therapy with warfarin was also initiated 24 hours after surgery and maintained at the target international normalized ratio (INR) of 2.0 to 2.5 for patients with a HeartMate II or DuraHeart, and 2.5 to 3.0 for those with a HeartWare, EVAHEART, or Jarvik2000. These different anticoagulation thresholds depend on the company's recommendation. Systemic heparinization was initiated when drainage became less than $100 \mathrm{~mL}$ in the previous 3 hours. We checked the activated partial thromboplastin time (aPTT) twice per day and managed to achieve a target aPTT (50-60 seconds) before achieving the target INR. Perioperative antibiotic therapy was routinely managed by experienced intensive care unit physicians. Postoperative mean blood pressure was usually controlled under $85 \mathrm{~mm} \mathrm{Hg}$.

\section{Definition and Management of Left Ventricular Assist Device-Related Adverse Events}

Preoperative neurologic examination using physical examination and brain computed tomography $(\mathrm{CT})$ was routinely performed in all patients. Postoperatively, if patients had any neurologic symptoms, including headache, dizziness, lightheadedness, syncope, or convulsion, we always performed brain CT without delay and referred to neurologists. A definition of stroke event has been described. ${ }^{4}$ A stroke event was defined as a neurologic symptom lasting for more than 24 hours with a compatible new lesion shown in brain CT scans. Stroke events are subdivided into ischemic and hemorrhagic stroke events. In the present study, hemorrhagic stroke included cerebral, cerebellum, and subarachnoid hemorrhaging, as well as subdural hematoma. Ischemic stroke was defined as a cerebral or cerebellum infarction without hemorrhagic lesions detected by brain CT scan. Headache or convulsion without any sustained neurologic deficit was not defined as an episode of stroke, unless patients had new lesions identified during neuroimaging. Furthermore, we defined the acute phase as within 90 days after LVAD implantation. ${ }^{4}$ Upon the occurrence of an LVAD-related stroke, treatment management was based on the discussion among experienced cardiologists, cardiovascular surgeons, neurosurgeons, and neurologists. The termination and reinitiation of antiplatelet or anticoagulation therapy was also managed after the discussion.

When our patients have any type of cerebral infarction, they are usually examined using CT angiography, and the indication of selective angiography or thrombectomy and the enforcement of antiplatelet and anticoagulant therapy are discussed with neurologists and neurosurgeons. On the other hand, when our patients have any type of intracranial hemorrhage lesion, antiplatelet and anticoagulant therapies are immediately ceased, normalization of INR using factor-IX or 
fresh-frozen plasma is started, and closed consecutive follow-up brain CT is performed until complete hemostasis is confirmed.

\section{Data Collection}

All patient data were obtained from the electronic medical records, including baseline characteristics, comorbidities, types of LVAD, and duration of LVAD implantation. Baseline characteristics (demographics, etiologies), laboratory values, hemodynamics, carotid Doppler, intraoperative parameters (cardiopulmonary bypass time, operation time, amount of blood products used, concomitant cardiac procedures), and postoperative complications were also collected from the electronic medical records and operative records. Postoperative hemodynamic parameters (central venous pressure, cardiac output, cardiac index) of Swan-Ganz catheter were collected from the intensive care unit medical charts. We further subdivided these hemodynamic parameters into 4 periods: postoperative 0 to 12 hours, 12 to 24 hours, 24 to 36 hours, and 36 to 48 hours, and the maximum and minimum digits of these hemodynamic parameters were manually extracted. Postoperative laboratory values were routinely evaluated every day until postoperative day 7. Unless patients had no serious complications, routine postoperative transthoracic echocardiography, the parameters of which were adopted in the present study, was performed within 4 weeks after LVAD implantation.

\section{Statistical Analysis}

All statistical analyses were performed using JMP version 12.0 (SAS Institute Inc, Cary, Calif) software. Categoric variables were summarized as frequencies and percentages and compared among groups using a chi-square or Fisher exact test. Continuous variables are summarized as the mean \pm standard deviation or median (interquartile range [IQR]) after testing for normality using the Shapiro-Wilk W test. All $P$ values for statistical analysis were 2 tailed.

The incidence rate of stroke was estimated using Kaplan-Meier curves, and multiple regression analysis was used to identify the risk factors for stroke within 90 days after LVAD implantation. Baseline characteristics, laboratory values, hemodynamics, intraoperative parameters (cardiopulmonary bypass time, operation time, amount of blood products used, concomitant cardiac procedures), postoperative parameters (maximum central venous pressure within postoperative 48 hours, minimum cardiac output, and cardiac index within postoperative 48 hours), complications (the amount of blood products used within postoperative 24 hours, blood loss within postoperative 24 hours, reexploration of bleeding, and requirement for continuous venovenous hemodialysis or right ventricular assist device), postoperative laboratory values, and parameters of postoperative transthoracic echocardiography were used as factors for this analysis. Initially, univariate analysis was applied, and then factors with a $P$ value less than .05 were considered for a multiple regression model to identify risk factors.

\section{RESULTS}

\section{Baseline Characteristics}

Patients' mortality with an LVAD at 90, 180, and 360 days after implantation was $5 \%, 8 \%$, and $13 \%$, respectively. A total of 40 patients $(25 \%)$ underwent heart transplantation with a mean waiting duration of $29 \pm 12$ months. The preoperative characteristics of the 158 enrolled patients are shown in Table 1 . The mean age was $43 \pm 14$ years, and 53 patients $(34 \%)$ were women. Because of the strict indications and eligibility requirements for placement on the heart transplantation list, comorbidity prevalence was low. The majority of these patients had idiopathic-dilated cardiomyopathy, and $97 \%$ of the entire cohort underwent
LVAD implantation as a bridge-to-transplantation therapy, because destination therapy was not approved in Japan at the end of December 2016.

\section{Incidence of Stroke and Comparison of Baseline Characteristics}

Of these 158 patients, $28(18 \%)$ developed stroke within 90 days of LVAD implantation. The rate of stroke at 7, 30, and 90 days was $4 \%, 7 \%$, and $18 \%$, respectively (Figure 1). The incidence of stroke within 90 days was more than half of that within 1 year, because the rate of stroke at 90,180 , and 360 days was $18 \%, 20 \%$, and $32 \%$, respectively, whereas that of stroke-related death was $5 \%, 5 \%$, and $10 \%$, respectively. Furthermore, hazard function of stroke showed a J-shaped curve with the highest risk immediately after LVAD implantation, which then increases again at postoperative 9 to 12 months (Figure 1, B). The details of stroke in the acute phase are summarized in Table E1. For those with stroke in the acute phase, cerebral infarction occurred in 8 patients, intracerebral hemorrhage occurred in 10 patients, subarachnoid hemorrhage occurred in 8 patients, and subdural hematoma occurred in 2 patients. Among patients with cerebral infarction, thrombectomy was performed in 2 patients, and 6 patients were managed by strengthening of the anticoagulation therapy. Every patient with cerebral infarction fully recovered without any permanent neurologic deficit. Among patients with intracerebral hemorrhage, all 10 patients were managed by infusion of factor-IX and fresh-frozen plasma transfusion. Three of them required decompressive craniectomy for salvage, although $5(50 \%)$ of 10 patients died, including 1 patient who underwent craniectomy. Meanwhile, LVAD pump thrombosis occurred in 2 patients in the acute phase. Of these, 1 patient (case 17) who was supported by the HeartMate II device and developed severe hemolysis with the maximum serum lactate dehydrogenase (LDH) level of $3400 \mathrm{U} / \mathrm{mL}$ at postoperative 36 days required emergency exchange of the HeartMate II LVAD and experienced cerebral infarction 16 days after the conversion. The other patient who was supported by the HeartMate II device also developed hemolysis with the maximum serum LDH level of $2000 \mathrm{U} / \mathrm{mL}$ at postoperative 41 days and required emergency conversion of the HeartMate II LVAD. The patient did not develop stroke during the follow-up period.

The comparison of patient characteristics, operative details, and postoperative echocardiographic parameters between patients with and without stroke in the acute phase is detailed in Table 1. Patients with stroke were significantly older, and stroke was more prevalent in women. Body surface area was significantly smaller in patients with stroke. There were no statistical differences among the etiologies, types of LVAD, preoperative hemodynamic, and laboratory valuables, except for the preoperative blood 
TABLE 1. Factors associated with stroke in acute phase $(n=158)$

\begin{tabular}{|c|c|c|c|c|}
\hline & $\begin{array}{c}\text { All } \\
(n=158)\end{array}$ & $\begin{array}{c}\text { Stroke (-) } \\
(\mathbf{n}=130)\end{array}$ & $\begin{array}{l}\text { Stroke }(+) \\
(\mathbf{n}=\mathbf{2 8})\end{array}$ & $P$ value \\
\hline Age at LVAD implantation (y) & $43 \pm 14$ & $42 \pm 14$ & $48 \pm 17$ & .013 \\
\hline Female, n (\%) & $53(34)$ & $39(30)$ & $14(50)$ & .049 \\
\hline Body surface area $\left(\mathrm{m}^{2}\right)$ & $1.6 \pm 0.2$ & $1.6 \pm 0.2$ & $1.5 \pm 0.2$ & .006 \\
\hline Body mass index $\left(\mathrm{kg} / \mathrm{m}^{2}\right)$ & $19.9 \pm 3.1$ & $20.1 \pm 3.1$ & $18.8 \pm 3.3$ & .096 \\
\hline \multicolumn{5}{|l|}{ Complications } \\
\hline Previous stroke, n (\%) & $10(6)$ & $8(6)$ & $2(7)$ & .855 \\
\hline Diabetes mellitus, n (\%) & $24(15)$ & $19(15)$ & $5(18)$ & 677 \\
\hline Hypertension, n (\%) & $12(8)$ & $8(6)$ & $4(14)$ & .144 \\
\hline \multicolumn{5}{|l|}{ INTERMACS profile } \\
\hline Profile I, n (\%) & $15(10)$ & $13(10)$ & $2(7)$ & 1.000 \\
\hline Profile II, n (\%) & $56(35)$ & $45(35)$ & $11(39)$ & .667 \\
\hline Profile III, n (\%) & $59(37)$ & $49(38)$ & $10(36)$ & 1.000 \\
\hline Profile IV, n $(\%)$ & $5(3)$ & $3(2)$ & $2(7)$ & .215 \\
\hline Bridge to bridge, $\mathrm{n}(\%)$ & $23(15)$ & $20(15)$ & $3(11)$ & .768 \\
\hline \multicolumn{5}{|l|}{ Cause } \\
\hline Ischemic cardiomyopathy, n (\%) & $20(13)$ & $14(11)$ & $6(21)$ & .128 \\
\hline Idiopathic dilated cardiomyopathy, $\mathrm{n}(\%)$ & $91(57)$ & $76(58)$ & $15(54)$ & 676 \\
\hline Hypertrophic cardiomyopathy, n (\%) & $22(14)$ & $19(15)$ & $3(11)$ & .767 \\
\hline \multicolumn{5}{|l|}{ Devices } \\
\hline HeartMate II (Abbott Medical, Abbott Park, Ill), n (\%) & $50(32)$ & $42(32)$ & $8(28)$ & .824 \\
\hline DuraHeart (Terumo Heart, Ann Arbor, Mich), n (\%) & $38(24)$ & $35(27)$ & $3(11)$ & .088 \\
\hline Jarvik2000 (Jarvik Heart, New York, NY), n (\%) & $33(20)$ & $24(19)$ & $9(32)$ & .125 \\
\hline EVAHEART (Sun Medical, Moriyama City, Japan), n (\%) & $23(15)$ & $18(14)$ & $5(18)$ & .562 \\
\hline HeartWare (Framingham, Mass), n (\%) & $14(9)$ & $11(8)$ & $3(11)$ & .715 \\
\hline \multicolumn{5}{|l|}{ Preoperative hemodynamics } \\
\hline Central venous pressure $(\mathrm{mm} \mathrm{Hg})$ & $8.1 \pm 4.9$ & $8.1 \pm 5.0$ & $8.5 \pm 4.8$ & .524 \\
\hline Mean pulmonary artery pressure $(\mathrm{mm} \mathrm{Hg})$ & $28.4 \pm 10.1$ & $28.3 \pm 10.1$ & $29.3 \pm 10.5$ & .647 \\
\hline Pulmonary wedge capillary pressure $(\mathrm{mm} \mathrm{Hg})$ & $20.5 \pm 8.4$ & $20.4 \pm 8.7$ & $21.2 \pm 7.4$ & .561 \\
\hline Cardiac index $\left(\mathrm{L} / \mathrm{min} / \mathrm{m}^{2}\right)$ & $2.1 \pm 0.6$ & $2.2 \pm 0.6$ & $2.1 \pm 0.5$ & .584 \\
\hline Inotropes, $\mathrm{n}(\%)$ & $138(88)$ & $115(88)$ & $23(85)$ & .745 \\
\hline Intra-aortic balloon pumping, $\mathrm{n}(\%)$ & $27(17)$ & $21(16)$ & $6(21)$ & .579 \\
\hline Intubation, $\mathrm{n}(\%)$ & $28(18)$ & $22(17)$ & $6(21)$ & .580 \\
\hline Use of extracorporeal LVAD, n (\%) & $29(19)$ & $6(4)$ & $2(7)$ & 622 \\
\hline \multicolumn{5}{|l|}{ Preoperative echocardiographic parameters } \\
\hline LVDd (mm) & $69.0 \pm 13.3$ & $70.1 \pm 13.1$ & $65.0 \pm 14.3$ & .061 \\
\hline LVDs (mm) & $63.5 \pm 14.0$ & $64.5 \pm 13.6$ & $60.0 \pm 15.7$ & .099 \\
\hline Left ventricular ejection fraction (LVEF, \%) & $20.7 \pm 8.8$ & $20.4 \pm 8.2$ & $22.2 \pm 11.3$ & 695 \\
\hline \multicolumn{5}{|l|}{ Preoperative laboratory valuables } \\
\hline Hemoglobin $(\mathrm{g} / \mathrm{dL})$ & $11.2(9.88-12.9)$ & $11.6(10.0-13.2)$ & $10.8(9.8-11.6)$ & .073 \\
\hline White blood cell count $\left(\times 1000 / \mathrm{mm}^{3}\right)$ & $6.6(4.9-8.0)$ & $6.6(4.9-8.0)$ & $6.5(4.8-7.8)$ & .973 \\
\hline $\mathrm{BUN}(\mathrm{mg} / \mathrm{dL})$ & $19(13-25)$ & $18(13-25)$ & $21(16-26)$ & .098 \\
\hline Creatinine $(\mathrm{mg} / \mathrm{dL})$ & $1.0(0.8-1.3)$ & $1.0(0.8-1.3)$ & $1.0(0.7-1.5)$ & 681 \\
\hline $\mathrm{eGFR}\left(\mathrm{mL} / \mathrm{min} / 1.73 \mathrm{~m}^{2}\right)$ & $58(44-82)$ & $58(45-83)$ & $59(36-76)$ & .495 \\
\hline Aspartate transaminase (IU/dL) & $27(21-36)$ & $27(21-36)$ & $26(20-36)$ & .573 \\
\hline Alanine transaminase (IU/dL) & $23(15-37)$ & $24(17-37)$ & $18(13-35)$ & .108 \\
\hline Total bilirubin $(\mathrm{mg} / \mathrm{dL})$ & $1.0(0.6-1.5)$ & $1.0(0.6-1.5)$ & $0.8(0.6-1.6)$ & .970 \\
\hline Albumin (mg/dL) & $3.8(3.2-4.1)$ & $3.8(3.2-4.2)$ & $3.7(2.9-4.1)$ & .400 \\
\hline LDH (IU/L) & $248(205-359)$ & $248(205-359)$ & $263(199-366)$ & .849 \\
\hline
\end{tabular}


TABLE 1. Continued

\begin{tabular}{|c|c|c|c|c|}
\hline & $\begin{array}{c}\text { All } \\
(n=158)\end{array}$ & $\begin{array}{c}\text { Stroke }(-) \\
(\mathbf{n}=130)\end{array}$ & $\begin{array}{l}\text { Stroke }(+) \\
(\mathbf{n}=\mathbf{2 8})\end{array}$ & $P$ value \\
\hline \multicolumn{5}{|l|}{ Operative valuables } \\
\hline Re-do surgery, n (\%) & $50(32)$ & $38(31)$ & $12(43)$ & .181 \\
\hline CPB time (min) & $150 \pm 62$ & $145 \pm 59$ & $174 \pm 69$ & .028 \\
\hline Aortic valve surgery, n (\%) & $10(6)$ & $7(6)$ & $3(11)$ & .375 \\
\hline \multicolumn{5}{|l|}{ Perioperative characteristics } \\
\hline Blood loss within $24 \mathrm{~h}(\mathrm{~mL})$ & $1360(964-1993)$ & $1415(976-2131)$ & $1101(882-1598)$ & .071 \\
\hline $\mathrm{RBC}$ transfusion in postoperative $24 \mathrm{~h}(\mathrm{~mL})$ & $483(233-960)$ & $538(250-1031)$ & $275(10-745)$ & .094 \\
\hline FFP transfusion in postoperative $24 \mathrm{~h}(\mathrm{~mL})$ & $775(477-1258)$ & $800(475-1257)$ & $660(488-1231)$ & .533 \\
\hline \multicolumn{5}{|l|}{ Postoperative echocardiographic parameters } \\
\hline Postoperative LVDd (mm) & $53.9 \pm 14.7$ & $49.6 \pm 14.7$ & $54.8 \pm 15.0$ & .218 \\
\hline Postoperative LVDs (mm) & $48.7 \pm 15.4$ & $43.9 \pm 15.4$ & $49.7 \pm 15.6$ & .207 \\
\hline Postoperative LVEF (\%) & $22.8 \pm 12.1$ & $21.8 \pm 11.9$ & $25.0 \pm 14.4$ & .441 \\
\hline
\end{tabular}

Blood loss within 24 hours, total amount of drainage tube within 24 hours. $L V A D$, Left ventricular assist device; INTERMACS, Interagency Registry for Mechanically Assisted Circulatory Support; $L V D d$, left ventricular end-diastolic dimension; $L V D s$, left ventricular end-systolic dimension; $L V E F$, left ventricular ejection fraction; $B U N$, blood urea nitrogen; $e G F R$, estimated glomerular filtration rate; $L D H$, lactate dehydrogenase; $C P B$, cardiopulmonary bypass; $R B C$, red blood cell; $F F P$, fresh-frozen plasma.

urea nitrogen levels, although there was a trend of the left ventricle being smaller in patients with stroke. As for the operative and postoperative parameters, patients with stroke had significantly longer cardiopulmonary bypass time. There was a trend of less blood products required by patients with stroke compared with patients without stroke, and the total amount of drainage tube during the first 24 days was significantly less in patients with stroke.

\section{Change of Laboratory and Hemodynamic Parameters}

We compared the change of serum coagulant factor during the first 2 weeks (prothrombin time international normalized ratio [PT-INR], aPTT, and serum LDH) between patients with and without stroke in the acute phase (Figure $2, A-C$ ). There were $57 \%$ and $62 \%$ of all patients whose PT-INR was in normal ranges at postoperative 7 days and 14 days, respectively. There was no significant difference in the change of PT-INR between patients with and without stroke throughout the first 2 weeks
(Figure 2, A). Although patients with stroke had higher aPTT levels at 2 and 3 postoperative days than patients without stroke, there was no statistical difference at 4 days postsurgery and thereafter (Figure 2, B). In contrast, patients with stroke in the acute phase had significantly higher LDH levels from the first postoperative day to 2 weeks postsurgery (Figure 2, C).

Figure 3 shows the change of postoperative minimum cardiac output (Figure 3,A) and minimum cardiac index (Figure 3,B) of Swan-Ganz catheter during the first 2 postoperative days. In the overall cohort, patients with stroke had significantly lower minimum cardiac output between 12 and 48 postoperative hours than patients without stroke (Figure 3,A). When we evaluated the minimum cardiac index, patients with stroke had a significantly lower cardiac index at 12 to 24 hours (Figure 3, B). Because our cohort included both axial and centrifugal LVADs, we subdivided LVADs into these 2 types (Figure 4). Seventeen patients with axial LVAD and 11 patients with centrifugal LVAD developed stroke in the
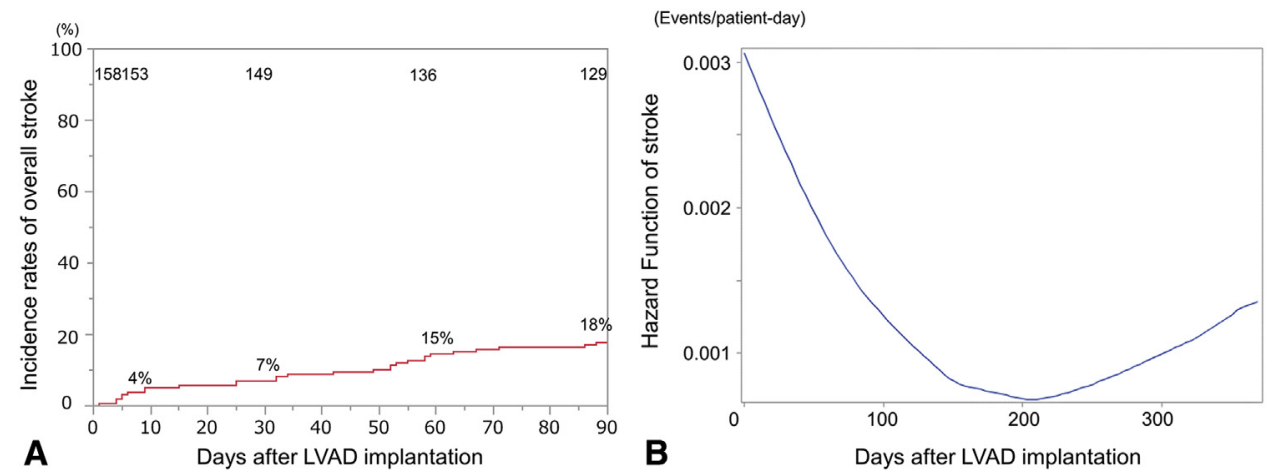

FIGURE 1. A, Incidence rates of overall stroke in the entire cohort. B, Hazard function of stroke after LVAD implantation. LVAD, Left ventricular assist device. 


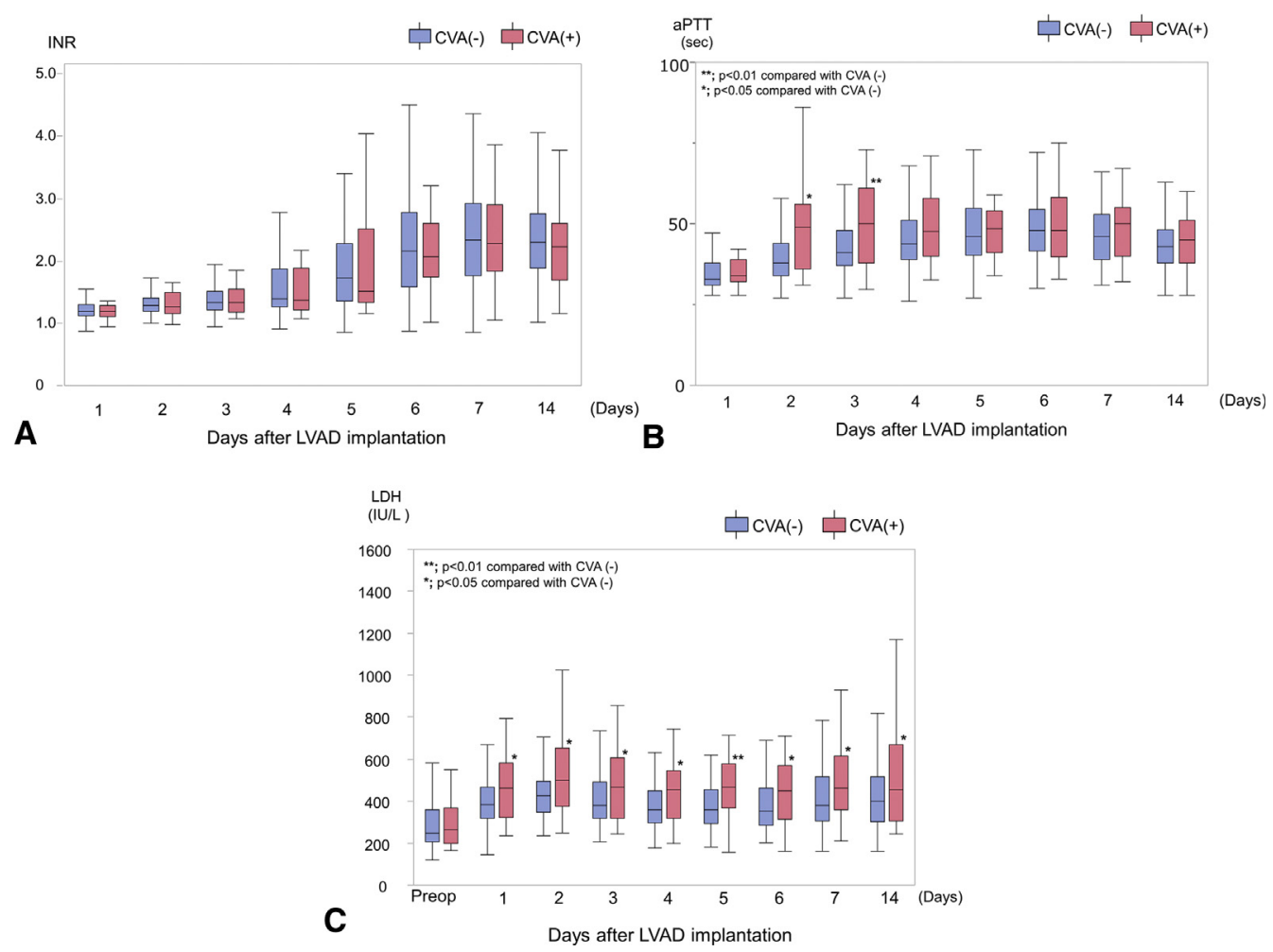

FIGURE 2. Postoperative course of (A) INR, (B) aPTT, and (C) serum LDH levels during the first postoperative 2 weeks. The horizontal line in the middle of each box indicates the median; the top and bottom borders of the box mark the 75th and 25th percentiles, respectively; and the whiskers mark the 90th and 10th percentiles. INR, International normalized ratio; $C V A$, cerebrovascular accident; $L V A D$, left ventricular assist device; $a P T T$, activated partial thromboplastin time; $L D H$, lactate dehydrogenase.

acute phase. In both types of LVADs, patients with stroke tended to have lower cardiac output and index than patients without stroke. The minimum cardiac output between 24 and 36 postoperative hours in patients with stroke was significantly lower than in patients without stroke (Figure 4, $A$ and $B$ ).

\section{Risk Factors for Stroke}

The risk factors for stroke in the acute phase are summarized in Table 2. Parameters with a statistically significant difference in comparison between patients with and without stroke in the acute phase were studied in the multivariate analysis. For postoperative minimum cardiac output, cardiac output during postoperative 12 to 24 hours was included because this period had the strongest odds ratio. Multivariate analysis revealed that low cardiac output (odds ratio, $0.25 ; 0.07-0.92 ; P=.024$ ) during postoperative 12 to 24 hours was the only independent risk factor for stroke in acute phase after LVAD implantation (Table 2). Age, female sex, body surface area, and cardiopulmonary
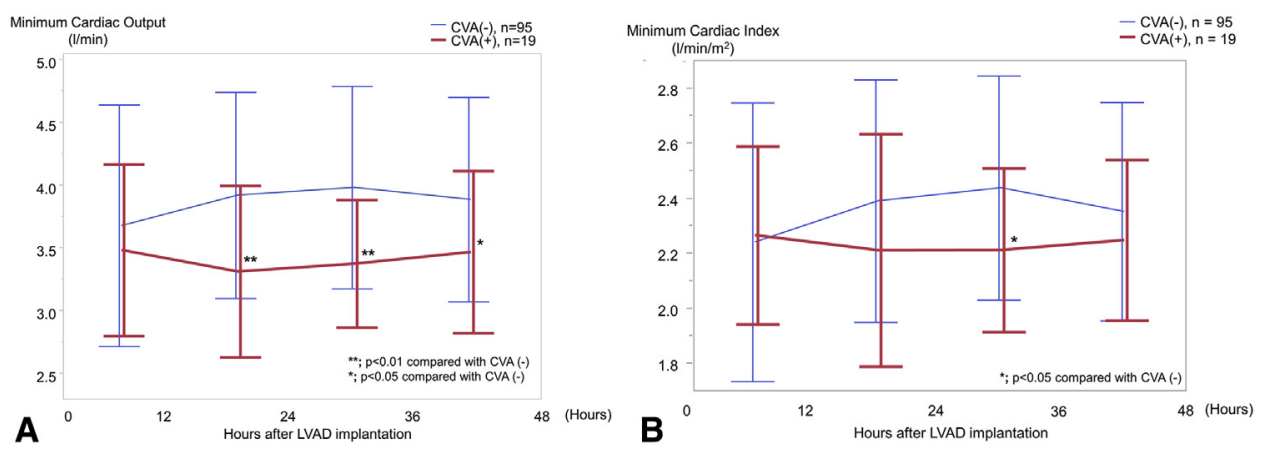

FIGURE 3. Postoperative course of (A) the minimum cardiac output in patients with stroke (red line) versus stroke-free patients (blue line) and (B) minimum cardiac index in patients with stroke (red line) versus stroke-free patients (blue line) after LVAD implantation. CVA, Cerebrovascular accident; $L V A D$, left ventricular assist device. 

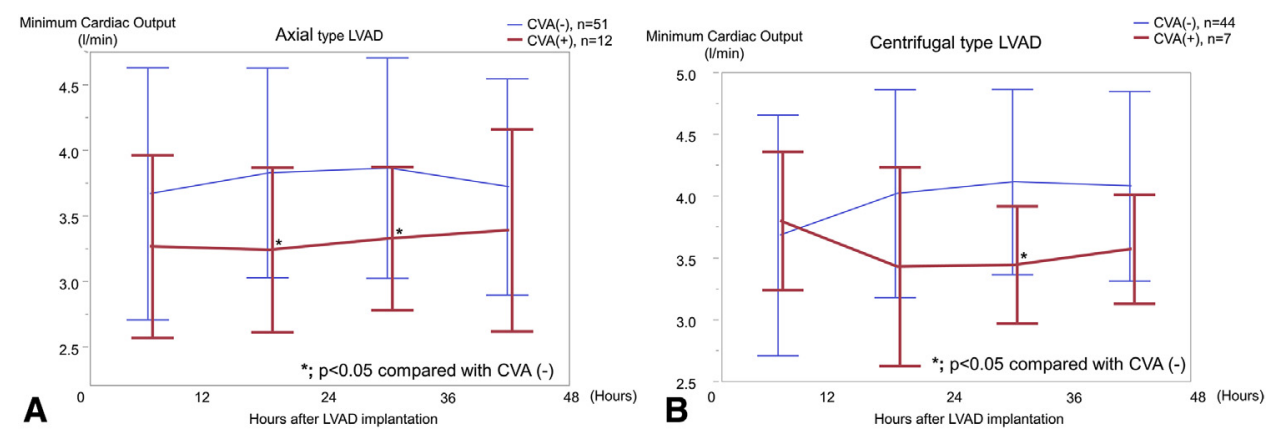

FIGURE 4. Postoperative course of (A) the minimum cardiac output in patients with stroke (red line) versus stroke-free patients (blue line) after axial type LVAD implantation and (B) the minimum cardiac output in patients with stroke (red line) versus stroke-free patients (blue line) after centrifugal type LVAD implantation. CVA, Cerebrovascular accident; $L V A D$, left ventricular assist device.

bypass time were not considered as risk factors for stroke in the acute phase.

\section{Relation Between Hemodynamic Parameters and Coagulant Factors}

Because the minimum cardiac output at postoperative 12 to 24 hours was the exclusive risk factor for stroke in the acute phase and serum LDH level was significantly higher in patients with stroke, we further evaluated the relationship between the minimum cardiac output at 12 to 24 hours and the serum LDH level at postoperative 3, 7, and 14 days (Figure 5, $A-C$ ). To eliminate the device bias, we evaluated the mentioned relationship in 50 patients with the HeartMate II device. Although there was no significant correlation between the minimum cardiac output and the serum LDH level until 7 days, a significant negative correlation was present at 14 days $(\mathrm{r}=-0.313, P=.03)$.

\section{DISCUSSION}

In the present study, stroke rate at 90 days, 180 days, and 1 year was $18 \%, 20 \%$, and $32 \%$, respectively. More than one half of stroke events within 1 year occurred within 90 days postoperatively, whereas the stroke rate from postoperative 90 to 180 days was only $2 \%$. Frontera and colleagues $^{5}$ reported that the risk for stroke after LVAD implantation showed a bimodal J-shaped curve, with the highest risk immediately after LVAD implantation, which then increased again at postoperative 9 to 12 months. The present study revealed a result similar to the previous study (Figure 1, B). The previous study and ours may imply that the cause of stroke could be divided into 2 phases: the acute phase and the chronic phase. We have also previously revealed that systemic bacteremia was an independent risk factor for hemorrhagic stroke in the chronic phase, whereas age was an independent risk factor for stroke in the acute phase. ${ }^{4,8}$ However, in our previous study, we could not include the data on the postoperative hemodynamic and laboratory parameters in the analysis. Therefore, in the previous study, it was not clear how to reduce the incidence of stroke in the acute phase.

In the analysis, PT-INR and aPTT during the first 2 weeks were almost similar, except for aPTT at 2 and 3 days, among patients with and without stroke. These results can imply that we cannot sufficiently prevent stroke in the acute phase only by meticulous management of anticoagulation with warfarin and heparinization. Instead, we revealed that patients with stroke in the acute phase had significantly higher serum LDH levels from postoperative day 1 to postoperative 2 weeks than patients without stroke. This result may indicate that stroke in the acute phase can be related to thrombotic or thrombolytic complications in the pump. Previous reports have noted that pump thrombosis often occurred in the acute phase after LVAD implantation, and evaluation of serum LDH levels provided good sensitivity and specificity to detect pump thrombosis. ${ }^{9,10}$ In detail, the hazard function of pump thrombosis in the

TABLE 2. Multivariable risk factors for stroke in acute phase $(n=158)$

\begin{tabular}{|c|c|c|c|}
\hline \multirow[b]{3}{*}{ Parameters } & \multicolumn{3}{|c|}{ Stroke } \\
\hline & \multirow{2}{*}{$\frac{\text { Univariate }}{P \text { value }}$} & \multicolumn{2}{|c|}{ Multivariate } \\
\hline & & OR $(95 \%$ CI $)$ & $P$ value \\
\hline Age at LVAD implantation (y) & .013 & $1.04(0.98-1.10)$ & .159 \\
\hline Female, n (\%) & .049 & $2.02(0.37-11.0)$ & .417 \\
\hline Body surface area $\left(0.1 \mathrm{~m}^{2}\right)$ & .006 & $1.18(0.74-1.89)$ & .485 \\
\hline Minimum cardiac output in postoperative $12-24 \mathrm{~h}(\mathrm{~L} / \mathrm{min})$ & .002 & $0.25(0.07-0.92)$ & .024 \\
\hline CPB time (min) & .028 & $1.00(0.99-1.01)$ & .630 \\
\hline
\end{tabular}

$O R$, Odds ratio; $C I$, confidence interval; $L V A D$, left ventricular assist device; $C P B$, cardiopulmonary bypass. 

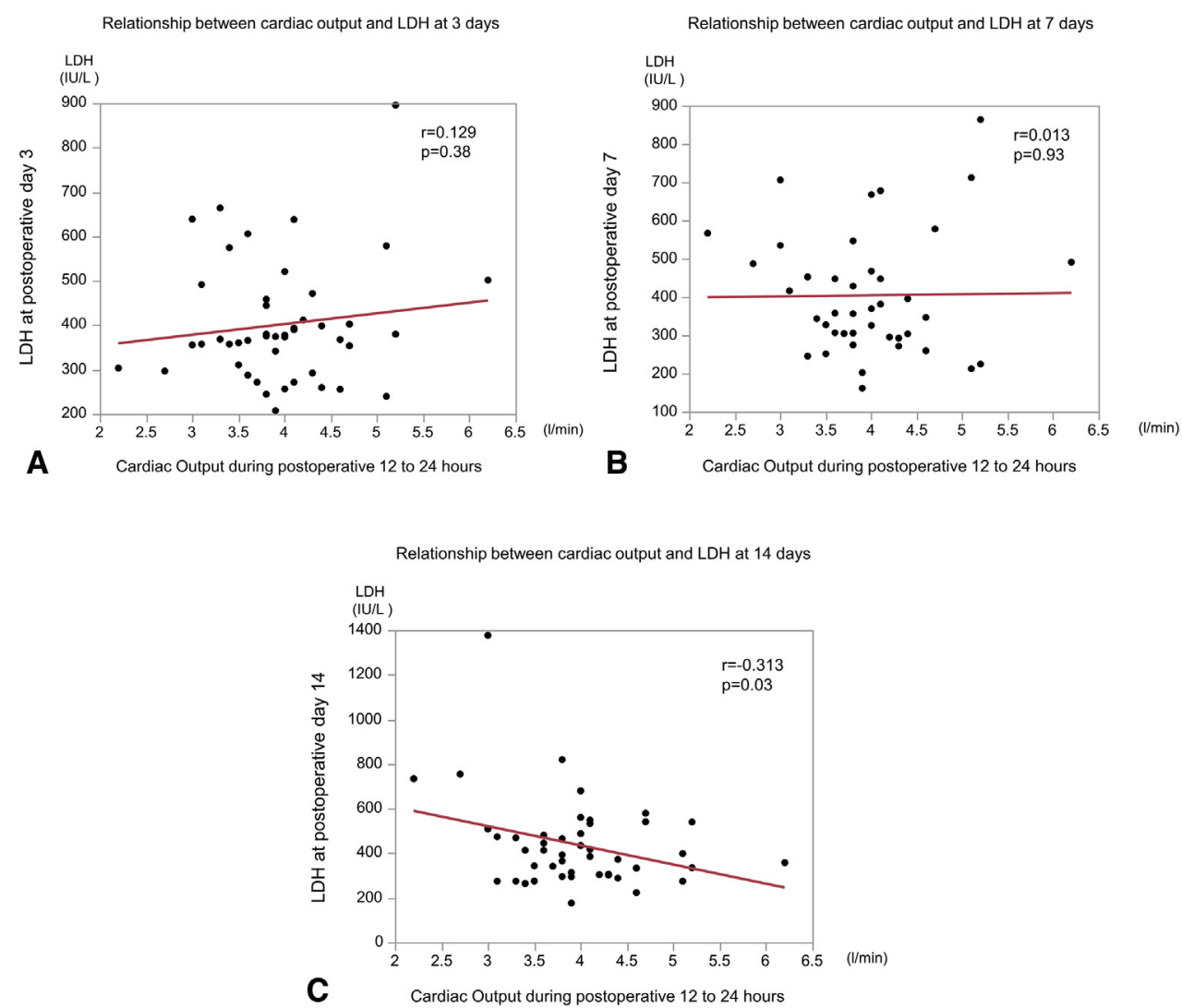

FIGURE 5. The relationship between the minimum cardiac output and the LDH (A) at 3 days, (B) 7 days, and (C) 2 weeks after implantation of the HeartMate II (Abbott Medical, Abbott Park, Ill). LDH, Lactate dehydrogenase.

HeartMate II device peaked at approximately 2 months after LVAD implantation, and marked elevation of LDH at 1 month after LVAD implantation was a predictor of subsequent pump thrombosis. ${ }^{10-12}$ Therefore, to prevent stroke in the acute phase, it is important to prevent LVAD thrombus formation during this period, although the mechanism and risk factor of thrombus formation in patients with LVADs were unclear. In the present study, we revealed that lower minimum cardiac output in the Swan-Ganz catheter, which is estimated to be almost the same as LVAD pump flow in the majority of patients, was the exclusive risk factor for stroke in the acute phase. To date, a negative correlation between the cardiac output and the incidence of stroke has not been reported. Several previous studies revealed predictors of stroke, including hypertension, female sex, and small body surface area, in the acute phase, although the detailed reasoning has not been discussed. ${ }^{12,13}$ All of these previously reported predictors, such as hypertension, female gender, and small body surface area, are also risk factors of low LVAD flow. Because experimental and fluid dynamic studies revealed that lower pump flow predisposes the pump to pump thrombosis (by shear stress and heat generation from pump rotor), ${ }^{14,15}$ we speculated that lower LVAD pump flow can precipitate subclinical pump thrombus and cause subsequent stroke in patients with small body surface area, including women, even though cardiac index was adequately maintained. Figure $3, B$, shows that patients had a higher risk for strokes, even though the cardiac index was maintained above 2.2. Therefore, we think that sufficient cardiac output or LVAD pump flow, not cardiac index, should be achieved to prevent stroke in the acute phase, particularly in patients with small body size. Blood pressure and afterload control have been considered important to prevent strokes. ${ }^{16-18}$ Because reduction of afterload increases LVAD pump flow, blood pressure control is also important to maintain adequate LVAD pump flow and prevent strokes.

To strengthen our hypothesis that lower LVAD pump flow can predispose the pump to subclinical pump thrombosis, which may cause strokes in the acute phase after LVAD implantation, we evaluated the correlation between cardiac output and serum LDH levels at 3, 7, and 14 days after LVAD implantation. Serum LDH levels, with LDH half-life depending on isozyme, seemed to be multifactorial, such as muscle injury during surgery especially in the postoperative super-acute phase. However, these intraoperative factors gradually declined, and thrombotic and thrombolytic factors became evident. Our analysis revealed a negative correlation between the 


\section{Seventh INTERMACS annual report: 15,000 patients and counting}
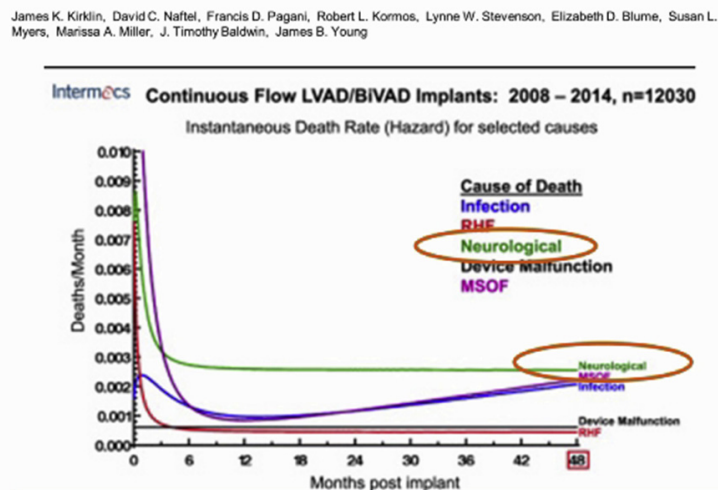

Stroke is one of major adverse events after LVAD implantation

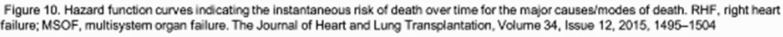

VIDEO 1. The video briefly summarizes the important findings of this study. Video available at: https://www.jtcvs.org/article/S0022-5223(18) 31765-3/fulltext.

perioperative cardiac output and the serum LDH levels at 14 days. These results indicate that sufficient LVAD pump flow may be important to prevent subclinical thrombosis formation and avoid strokes in the acute phase after implantation. The threshold of "sufficient flow" in each device should be further evaluated in future studies with large numbers of patients.

\section{Study Limitations}

Our study has several limitations. First, this study is a retrospective study in a single center, and the number of patients with stroke in the acute phase is inevitably limited. Second, 5 different types of LVADs were included, because the available devices varied during the study period. Although there were no statistical differences among the various LVADs used, the numbers of each device were further limited, and the analysis might have been underpowered. Third, although we defined stroke in the acute phase as stroke that occurred within postoperative 90 days, we cannot conclude that all stroke events were caused by embolic events. Therefore, we could not completely eliminate cases of stroke caused by other factors such as spontaneous bleeding of the cerebral artery. Finally, our analysis could overlook some stroke events, because we did not perform brain CT in patients without headache or any neurologic symptoms.

\section{CONCLUSIONS}

More than one half of stroke events within 1 year after LVAD implantation occurred within 90 days, and stroke in the acute phase may be related to subclinical pump thrombus formation due to low and insufficient LVAD flow. Our study suggested that maintenance of sufficient LVAD flow, as well as anticoagulation therapy, plays a critical role in prevention of stroke in the acute phase after LVAD implantation (Video 1).

\section{Conflict of Interest Statement}

Authors have nothing to disclose with regard to commercial support.

\section{References}

1. Starling RC, Naka Y, Boyle AJ, Gonzalez-Stawinski G, John R, Jorde U, et al. Results of the post-U.S. Food and Drug Administration-approval study with a continuous flow left ventricular assist device as a bridge to heart transplantation: a prospective study using the INTERMACS (Interagency Registry for Mechanically Assisted Circulatory Support). J Am Coll Cardiol. 2011;57: 1890-8.

2. Toda K, Sawa Y. Clinical management for complications related to implantable LVAD use. Gen Thorac Cardiovasc Surg. 2015;63:1-7.

3. Kirklin JK, Naftel DC, Pagani FD, Kormos RL, Stevenson LW, Blume ED, et al. Seventh INTERMACS annual report: 15,000 patients and counting. J Heart Lung Transplant. 2015;34:1495-504.

4. Yoshioka D, Sakaniwa R, Toda K, Samura T, Saito S, Kashiyama N, et al Relationship between bacteremia and hemorrhagic stroke in patients with continuous-flow left ventricular assist device. Circ J. 2018;82:448-56.

5. Frontera JA, Starling R, Cho SM, Nowacki AS, Uchino K, Hussain MS, et al Risk factors, mortality, and timing of ischemic and hemorrhagic stroke with left ventricular assist devices. J Heart Lung Transplant. 2017;36:673-83.

6. Nishi H, Toda K, Miyagawa S, Yoshikawa Y, Fukushima S, Saito S, et al. Initial experience in Japan with HeartWare ventricular assist system. J Artif Organs. 2014; 17:149-56

7. Yoshioka D, Matsumiya G, Toda K, Sakaguchi T, Yoshikawa Y, Saito S, et al. Clinical results with Jarvik 2000 axial flow left ventricular assist device: Osaka University Experience. J Artif Organs. 2014;17:308-14.

8. Yoshioka D, Okazaki S, Toda K, Murase S, Saito S, Domae K, et al. Prevalence of cerebral microbleeds in patients with continuous-flow left ventricular assist devices. J Am Heart Assoc. 2017;6.

9. Uriel N, Morrison KA, Garan AR, Kato TS, Yuzefpolskaya M, Latif F, et al. Development of a novel echocardiography ramp test for speed optimization and diagnosis of device thrombosis in continuous-flow left ventricular assist devices: the Columbia ramp study. J Am Coll Cardiol. 2012;60:1764-75.

10. Cowger JA, Romano MA, Shah P, Shah N, Mehta V, Haft JW, et al. Hemolysis: a harbinger of adverse outcome after left ventricular assist device implant. J Heart Lung Transplant. 2014;33:35-43.

11. Kirklin JK, Naftel DC, Kormos RL, Pagani FD, Myers SL, Stevenson LW, et al Interagency Registry for Mechanically Assisted Circulatory Support (INTERMACS) analysis of pump thrombosis in the HeartMate II left ventricular assist device. J Heart Lung Transplant. 2014;33:12-22.

12. Kirklin JK, Naftel DC, Pagani FD, Kormos RL, Myers S, Acker MA, et al. Pump thrombosis in the Thoratec HeartMate II device: an update analysis of the INTERMACS Registry. J Heart Lung Transplant. 2015; 34:1515-26.

13. Komoda T, Drews T, Hetzer R, Lehmkuhl HB. Lower body surface area is highly related to mortality due to stroke or systemic bleeding in patients receiving an axial flow blood pump as a left ventricular assist device. Eur J Cardiothorac Surg. 2013;43:1036-42.

14. Birschmann I, Dittrich M, Eller T, Wiegmann B, Reininger AJ, Budde U, et al Ambient hemolysis and activation of coagulation is different between HeartMate II and HeartWare left ventricular assist devices. J Heart Lung Transplant. 2014; 33:80-7.

15. Uriel N, Han J, Morrison KA, Nahumi N, Yuzefpolskaya M, Garan AR, et al Device thrombosis in HeartMate II continuous-flow left ventricular assist devices: a multifactorial phenomenon. J Heart Lung Transplant. 2014;33:51-9.

16. Lampert BC, Eckert C, Weaver S, Scanlon A, Lockard K, Allen C, et al. Blood pressure control in continuous flow left ventricular assist devices: efficacy and impact on adverse events. Ann Thorac Surg. 2014;97:139-46.

17. DeVore AD, Stewart GC. The risk of stroke on left ventricular assist device support: steady gains or stalled progress? JACC Heart Fail. 2017;5:712-4.

18. Maltais S, Kilic A, Nathan S, Keebler M, Emani S, Ransom J, et al. PREVENtion of HeartMate II Pump Thrombosis Through Clinical Management: The PREVENT multi-center study. J Heart Lung Transplant. 2017;36:1-12.

Key Words: left ventricular assist device, low cardiac output, pump thrombosis, stroke 
TABLE E1. Patients with stroke

\begin{tabular}{|c|c|c|c|c|c|c|c|c|c|c|}
\hline Case & $\begin{array}{c}\text { Age } \\
(y)\end{array}$ & Sex & $\begin{array}{l}\text { Type of } \\
\text { device }\end{array}$ & $\begin{array}{c}\text { Days } \\
\text { from } \\
\text { surgery } \\
\text { to CVA }\end{array}$ & $\begin{array}{c}\text { LDH } \\
\text { just } \\
\text { before } \\
\text { stroke } \\
\text { (IU/L) }\end{array}$ & $\begin{array}{c}\text { PT-INR } \\
\text { just } \\
\text { before } \\
\text { stroke }\end{array}$ & Type of CVA & $\begin{array}{l}\text { Location } \\
\text { and size }\end{array}$ & $\begin{array}{l}\text { Neurologic } \\
\text { symptom }\end{array}$ & $\begin{array}{l}\text { Neurologic } \\
\text { outcome }\end{array}$ \\
\hline 1 & 65 & $\mathrm{~F}$ & Jarvik2000 & 1 & 710 & 1.26 & Hemorrhagic infarction & Left frontal lobe & None & Aphasia \\
\hline 2 & 49 & M & DuraHeart & 4 & 319 & 1.13 & Cerebral infarction & Right cerebellar & Dysarthria & Full recovery \\
\hline 3 & 61 & $\mathrm{~F}$ & Jarvik2000 & 4 & 622 & 1.25 & Cerebral infarction & Right basal ganglia & Coma & Full recovery \\
\hline 4 & 20 & M & EVAHEART & 5 & 534 & 1.48 & Subarachnoid hemorrhage & Left frontal lobe & $\begin{array}{l}\text { Disordered } \\
\text { consciousness }\end{array}$ & Full recovery \\
\hline 5 & 59 & $\mathrm{~F}$ & Jarvik2000 & 5 & 1488 & 2.02 & Subcortical hemorrhage & $\begin{array}{l}\text { Right frontal lobe, } \\
\quad 40 \times 55 \times 60 \mathrm{~mm}\end{array}$ & Headache & Full recovery \\
\hline 6 & 12 & $\mathrm{~F}$ & Jarvik2000 & 6 & 603 & 1.87 & Subcortical hemorrhage & Right occipital lobe & Headache & Full recovery \\
\hline 7 & 61 & M & HeartMate II & 9 & 441 & 1.81 & Subcortical hemorrhage & $\begin{array}{l}\text { Right frontal lobe, } \\
40 \times 55 \times 60 \mathrm{~mm}\end{array}$ & $\begin{array}{l}\text { Lose } \\
\text { consciousness }\end{array}$ & Dead \\
\hline 8 & 61 & $\mathrm{~F}$ & HeartMate II & 9 & 632 & 3.39 & Left frontal lobe SDH & Left frontal lobe & Dysarthria & Full recovery \\
\hline 9 & 64 & $\mathrm{~F}$ & HeartMate II & 15 & 756 & 1.83 & Subcortical hemorrhage & Right frontal lobe & Coma & Dead \\
\hline 10 & 47 & $\mathrm{~F}$ & HeartWare & 25 & 198 & 2.20 & Subcortical hemorrhage & $\begin{array}{l}\text { Left frontal lobe, } \\
\quad 80 \times 55 \times 80 \mathrm{~mm}\end{array}$ & Anisocoria & Dead \\
\hline 11 & 61 & $\mathrm{~F}$ & Jarvik2000 & 25 & 495 & 3.02 & Subcortical hemorrhage & $\begin{array}{l}\text { Left cerebellar, } \\
\qquad 40 \times 55 \times 60 \mathrm{~mm}\end{array}$ & Headache & Full recovery \\
\hline 12 & 46 & $\mathrm{~F}$ & Jarvik2000 & 32 & 375 & 1.94 & Subarachnoid hemorrhage & $\begin{array}{l}\text { Anterior medulla } \\
\text { oblongata }\end{array}$ & Headache & Full recovery \\
\hline 13 & 50 & M & HeartMate II & 32 & 445 & 2.10 & Subcortical hemorrhage & $\begin{array}{l}\text { Right temporal lobe, } \\
\quad 78 \times 49 \times 70 \mathrm{~mm}\end{array}$ & Headache & Dead \\
\hline 14 & 55 & M & EVAHEART & 34 & 192 & 1.81 & Cerebral infarction & Left parietal lobe & Headache & Full recovery \\
\hline 15 & 59 & M & DuraHeart & 42 & 265 & 2.16 & Right frontal lobe SDH & Right frontal lobe & Headache & Full recovery \\
\hline 16 & 11 & M & HeartWare & 49 & 298 & 2.42 & Cerebral infarction & Right temporal lobe & Hemiplegia & Full recovery \\
\hline 17 & 64 & $\mathrm{~F}$ & HeartMate II & 52 & 417 & 1.72 & Subarachnoid hemorrhage & Frontal lobe & Seizures & Full recovery \\
\hline 18 & 53 & $\mathrm{~F}$ & EVAHEART & 52 & 243 & 2.43 & Cerebral infarction & Left thalamus & $\begin{array}{l}\text { Aphasia, } \\
\text { Hemiplegia }\end{array}$ & Full recovery \\
\hline 19 & 51 & M & EVAHEART & 53 & 265 & 2.74 & Cerebral infarction & Left basal ganglia & $\begin{array}{l}\text { Disordered } \\
\text { consciousness }\end{array}$ & Full recovery \\
\hline 20 & 62 & M & HeartMate II & 55 & 405 & 1.83 & Subcortical hemorrhage & $\begin{array}{l}\text { Right frontal lobe, } \\
40 \times 55 \times 60 \mathrm{~mm}\end{array}$ & Coma & Dead \\
\hline 21 & 13 & $\mathrm{~F}$ & HeartWare & 58 & 720 & 1.70 & Subarachnoid hemorrhage & Left frontal lobe & $\begin{array}{l}\text { Lose } \\
\text { consciousness }\end{array}$ & Full recovery \\
\hline 22 & 35 & $\mathrm{~F}$ & EVAHEART & 58 & 177 & 1.67 & Cerebral infarction & Left temporal lobe & $\begin{array}{l}\text { Aphasia, } \\
\text { Hemiplegia }\end{array}$ & Full recovery \\
\hline 23 & 36 & M & DuraHeart & 59 & 241 & 2.13 & Subarachnoid hemorrhage & Left temporal lobe & None & Full recovery \\
\hline 24 & 49 & M & HeartMate II & 63 & 573 & 1.4 & Subcortical hemorrhage & Right frontal lobe & Hemiplegia & Hemiplegia \\
\hline 25 & 44 & $\mathrm{~F}$ & Jarvik2000 & 67 & 346 & 1.78 & Subarachnoid hemorrhage & Left temporal lobe & $\begin{array}{l}\text { Visual field } \\
\text { defect }\end{array}$ & Full recovery \\
\hline 26 & 56 & M & Jarvik2000 & 71 & 360 & 2.81 & Subcortical hemorrhage & Left frontal lobe & $\begin{array}{l}\text { Lose } \\
\text { consciousness }\end{array}$ & Dead \\
\hline 27 & 55 & M & Jarvik2000 & 86 & 554 & 2.08 & Subarachnoid hemorrhage & Right frontal lobe & $\begin{array}{l}\text { Lose } \\
\text { consciousness }\end{array}$ & Full recovery \\
\hline 28 & 31 & M & HeartMate II & 88 & 298 & 2.85 & Subcortical hemorrhage & Right frontal lobe & Headache & Full recovery \\
\hline
\end{tabular}

CVA, Cerebrovascular accident; $L D H$, lactate dehydrogenase; $P T-I N R$, prothrombin time international normalized ratio; $S D H$, subdural hematoma. 